\title{
POTENTIAL OF C-BAND HYBRID POLARIMETRIC RISAT-1 SAR DATA FOR WHEAT CROP HEIGHT ESTIMATION
}

\author{
Hari Shanker Srivastava * and Thota Sivasankar \\ Indian Institute of Remote Sensing (ISRO), Dehradun - 248001, India -( $\underline{\text { hari.isro, siva.iirs)@ gmail.com }}$
}

Commission III, WG III/10

KEY WORDS: Hybrid polarimetry, RISAT-1 SAR, Wheat, Plant height, $m-\chi$ decomposition

\begin{abstract}
:
The advancement of hybrid polarimetry and the launch of Radar Imaging SATellite-1 (RISAT-1), the first Earth observing satellite with capability to acquire hybrid polarimetric SAR data, have given opportunity to explore the potentials of this data for various geospatial applications. Among these, crop growth monitoring and soil moisture estimation is one of the important application for proper irrigation management, yield forecasting and crop insurance systems. The objective of this paper is to provide a better understanding of the sensitivity of RISAT-1 hybrid polarimetric SAR data for wheat height. However, backscattering coefficients have shown less sensitive towards wheat height, polarimetric parameters such as Stokes parameters and scattering decomposition parameters have shown higher sensitivity. The analysis observed the highest correlation coefficient $\left(|\mathrm{r}|\right.$ ) and lowest RMSE (in $\mathrm{m}$ ) for $\mathrm{g}_{3}$ with 0.61 and 0.18 respectively followed by odd bounce with 0.59 and 0.2 respectively.
\end{abstract}

\section{INTRODUCTION}

Wheat is one of the world's most commonly consumed cereal grains and is grown on larger area than any other food crop (Curtis et al., 2002). However, the production of wheat is not adequate as per the demand. Therefore, a precise and accurate wheat crop growth monitoring system is necessary for proper irrigation management, yield forecasting and crop insurance systems.

Crop biophysical parameters (such are height, biomass, leaf area index and water content) dynamics during crop growing season are a vital input for assessing crop status and yield estimation (Doraiswamy et al., 2003; Patel et al., 2006). Since these parameters are highly dynamic in spatial as well as temporal, ground-based field measurement is labour intensive and time consuming. Remotely sensed data has become primary source for this purpose by providing high spatial and temporal images from local to global scales. The SAR technology which uses microwave region of the electromagnetic spectrum has emerged as an alternative by providing high-spatial resolution data in all-weather and day-night conditions. The return signal characteristics are highly sensitive towards geometrical, structural and dielectric properties of various crop components (Henderson and Lewis, 1998). SAR sensor parameters such are frequency, polarization and incidence angle have to be chosen properly to enhance the sensitivity of return signal for a specific application (Srivastava et al., 2008). Target parameters retrieval using SAR data is still a challenge due to its complex relationship of sensor as well as target parameters. Numerous studies have shown the potentials of SAR data for wide range of agricultural crop applications (Sivasankar et al., 2018; Steele-Dunne et al., 2017).
Backscattering coefficients observed at high incidence angles have shown higher sensitivity towards plant water content and less sensitive towards plant height (Kumar et al., 2018). The potentials to retrieval plant height using other SAR technologies viz. InSAR (Srivastava et al., 2006) and PolInSAR (Erten et al., 2016) has also been investigated. However, these techniques have found to be more suitable for accurate plant height estimation than backscattering coefficient. Polarimetric parameters such are even bounce, odd bounce and volume component have shown better performance for crop biophysical parameters retrieval in comparison to backscattering coefficients (Srivastava et al., 2018a). Volume component, which is proportional to un-polarized component of return signal, was considered as the return signal component from vegetation cover. However, Srivastava et al., (2018b) observed that the underneath soil moisture also influence volume component depending on the soil exposure (1-LAI).

The recent advancement of hybrid polarimetry, which illuminates circular polarization and receives two orthogonal coherent linear polarizations commonly using horizontal and vertical, has several advantages over traditional linear polarimetry. After the successful launch of RISAT-1, real hybrid polarimetric SAR data over Earth has made available to remote sensing community. Several studies have been carried to assess the potentials of hybrid polarimetric SAR data for various geo-spatial applications (Chakraborty et al., 2013). This study is focused to analyze the potentials of RISAT-1 hybrid polarimetric SAR data (FRS-1 mode) for wheat height estimation. The details of study area and datasets used (satellite data and ground-truth data) are given in Section-2. This study results have been discussed in Section-3. The conclusions of this study are made in Section-4.

*Corresponding author 


\section{MATERIALS AND METHODS}

\subsection{Study area and satellite data}

Present study has used three RISAT-1 hybrid polarimetric SAR datasets (FRS-1 mode) were acquired at $\sim 31^{\circ}$ incidence angle over parts of Bharatpur and Mathura districts located in Rajasthan and Uttar Pradesh states respectively in India during Jan - Mar 2016 (25 January 2016; 19 February 2016; 15 March 2016). The study area is flat terrain and usually dominated with wheat and mustard crops during rabi season (geographical location is shown in Fig 1). The Yamuna canal passing through the Mathura district enables the irrigation water for crops in this area. Since the sensor parameters are same during the both campaigns, the changes in the sensitivity of parameters generated from RISAT-1 hybrid polarimetric SAR data towards wheat height were considered it as caused by the crop growth. The wheat crop growth in the study area was at stem elongation, milking and ripening stages on 25 January, 19 February, 15 March respectively.

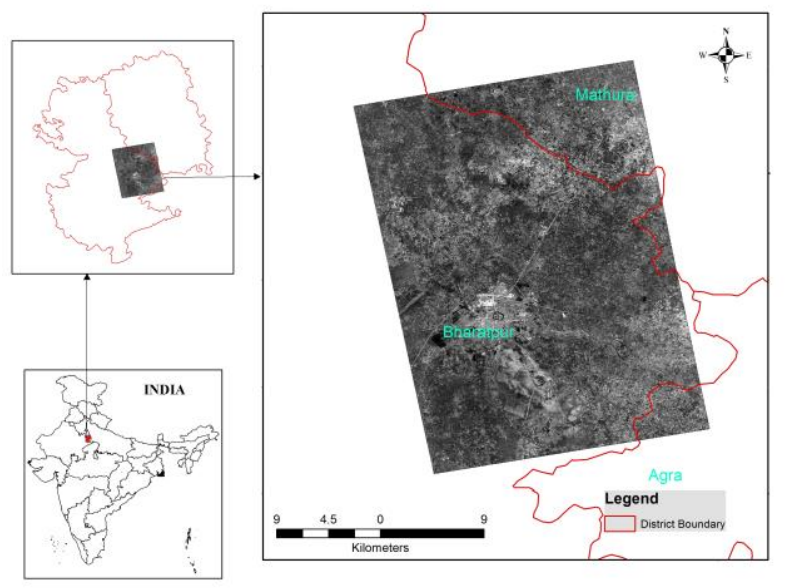

Figure 1. Location of the study area

\subsection{Ground-truth data collection}

Patel and Srivastava (2013) strategy has been used for field data collection in synchrony to the satellite passes by accounting the characteristic-fading phenomenon of SAR signal. The farmers' fields of greater than $630 \mathrm{~m}^{2}$ were considered to attain the error of $10 \%$ on the signal amplitude at $95 \%$ confidence interval. The in-situ wheat plant height from 84 of such wheat fields were measured during the campaigns (among these samples 28, 31 and 25 were collected during stem elongation, milking and ripening stages respectively). Crop height was measured by averaging 5 replications at each field, four on corners and one at center. The field photos taken during campaigns showing crop growth in a field and in-situ data collection are given in Figure 2. to illustrate the change in crop structure due to the crop growth. The box-chart plot of wheat height during the campaigns is given in Fig 3.

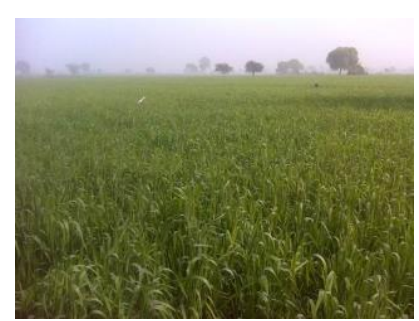

(a)

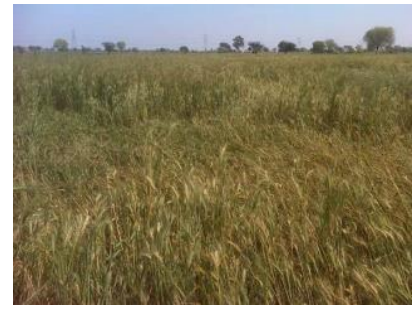

(c)

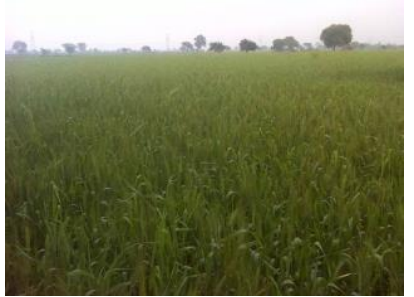

(b)

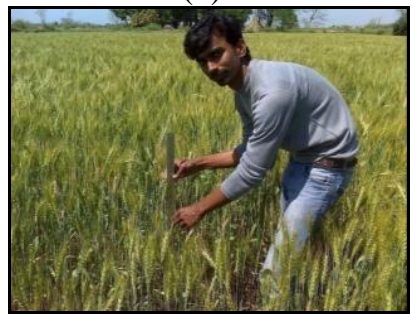

(d)
Figure 2. Ground-based photographs of a wheat field taken on (a) 25 January 2016; (b) 19 February 2016; (c) 15 March 2016 and in-situ data collection

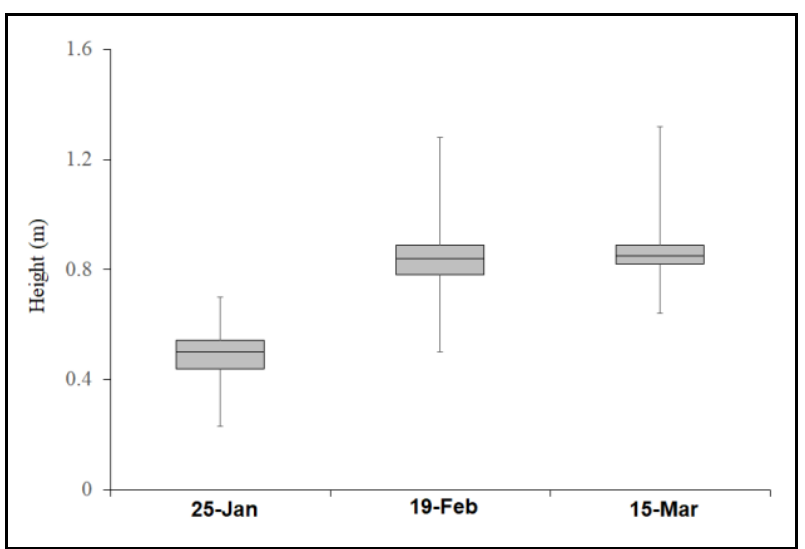

Figure 3. Box chart of in-situ wheat plant height

\subsection{Hybrid polarimetric parameters}

The RISAT-1 hybrid polarimetric SAR datasets were radiometrically calibrated using the following equation as given by Mishra et al., (2015).

$$
\sigma_{(d B)}^{o}=10 \times \log _{10}\left(\frac{D N_{i}^{2}}{C_{i i}}\right)+10 \times \log _{10}\left(\frac{\sin \left(\theta_{i}\right)}{\sin \left(\theta_{\text {center }}\right)}\right)
$$

Where, $\mathrm{C}_{\mathrm{ii}}$ is calibration constant, $\theta_{\mathrm{i}}$ represents incidence angle of $\mathrm{i}^{\text {th }}$ pixel and $\theta_{\text {center }}$ is center incidence angle.

Stokes defined a set of four parameters based on two linear orthogonal polarization signals (horizontal and vertical) to describe the polarization state of the electromagnetic signal. The Stokes parameters were generated using following equation. 


$$
g=\left[\begin{array}{l}
g_{0} \\
g_{1} \\
g_{2} \\
g_{3}
\end{array}\right]=\left[\begin{array}{c}
\left\langle\left|E_{R H}\right|^{2}+\left|E_{R V}\right|^{2}\right\rangle \\
\left\langle\left|E_{R H}\right|^{2}-\left|E_{R V}\right|^{2}\right\rangle \\
2 \times \operatorname{Re}\left\langle E_{R H} E_{R V}^{*}\right\rangle \\
-2 \times \operatorname{Im}\left\langle E_{R H} E_{R V}^{*}\right\rangle
\end{array}\right]
$$

Here, $\mathrm{E}$ is the complex backscattered electric field, $|\ldots|$ denotes absolute value, $\langle\ldots\rangle$ represents average, $*$ indicates complex conjugate and Re \&Im denotes the real and imaginary parts of the complex respectively.

Raney et al., (2012) developed $m-\chi$ decomposition technique based on " $m$ " and degree of circularity $(\chi)$. The relationship of degree of circularity and the Stokes parameters is given as,

$\sin 2 \chi=\frac{g_{3}}{\left(m \times g_{0}\right)}$

Where " $\mathrm{m}$ " represents the degree of polarization and can be derived using following equation:

$$
m=\frac{\sqrt{g_{1}^{2}+g_{2}^{2}+g_{3}^{2}}}{g_{0}}
$$

The parameters generated using $\mathrm{m}-\chi$ decomposition is expressed as,

$$
\begin{aligned}
& \text { Volume }=\sqrt{(1-m) \times g_{0}} \\
& \text { Even }=\sqrt{m \times g_{0}(1-\sin 2 \chi / 2)} \\
& \text { Odd }=\sqrt{m \times g_{0}(1+\sin 2 \chi / 2)}
\end{aligned}
$$

$\mathrm{m}-\chi$ decomposition technique derived parameters have shown better performance for crop biophysical parameters retrieval than $\mathrm{m}-\delta$ and $\mathrm{m}-\alpha$ decomposition techniques (Srivastava et al., 2018a).

\section{RESULTS AND DISCUSSION}

Regression analysis has been carried to analyze the potentials of hybrid polarimetric parameters generated from RISAT-1 SAR data for wheat height retrieval. The hybrid polarimetric parameters such are backscattering coefficients $\left(\sigma^{\circ} \mathrm{RH}\right.$ and $\left.\sigma^{\circ} \mathrm{RV}\right)$, Stokes parameters $\left(\mathrm{g}_{0}, \mathrm{~g}_{1}, \mathrm{~g}_{2}\right.$ and $\left.\mathrm{g}_{3}\right)$ and scattering decomposition (even bounce, odd bounce and volume component) have been considered in this study.

\subsection{Sensitivity analysis of backscattering coefficients}

The scatterplots of multi-temporal backscattering coefficients $\left(\sigma_{\mathrm{RH}}^{\circ}\right.$ and $\left.\sigma_{\mathrm{RV}}^{\circ}\right)$ with plant height has been given in Fig 4. The correlation coefficient and RMSE (in $\mathrm{m}$ ) of 0.32 and 0.41 respectively observed for $\mathrm{RH}$ backscatter; whereas 0.16 and 0.94 observed for RV backscatter. This study observed the absorption of illuminated microwave signal by the wheat similar to some of the previous studies (Paloscia et al., 2014; Macelloni et al., 2001). It is observed that initially the backscatter decreases with increase in plant height, whereas during ripening stage the plant height remain high but the backscatter increases than in mature stage. This indicates that the backscatter is less sensitive towards plant height and is more sensitive towards the plant water content. These results support the analysis carried by Kumar et al., (2018).

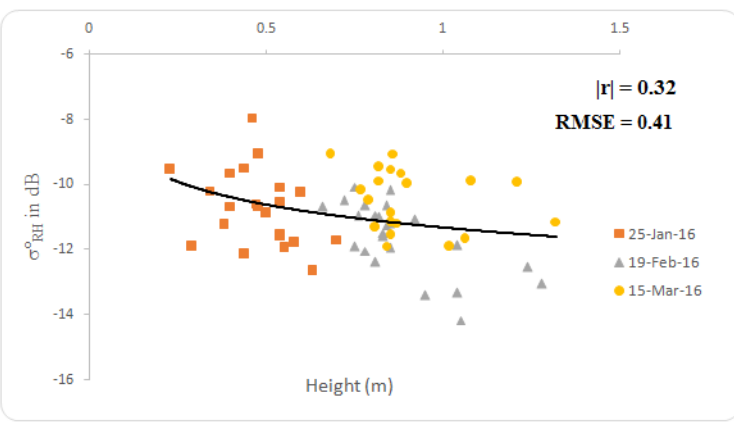

(a)

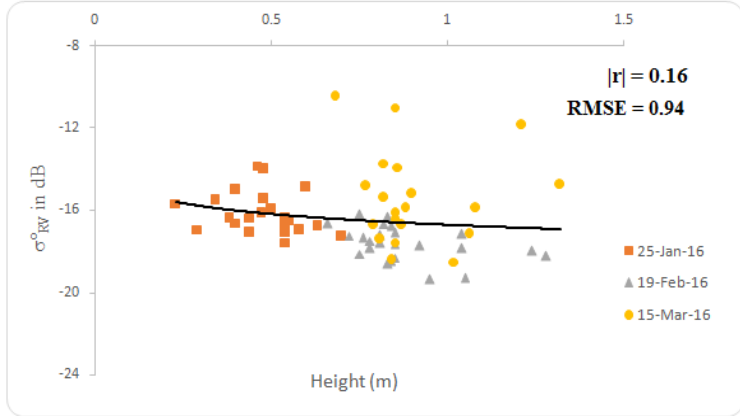

(b)

Figure 4. Scatterplot of backscattering coefficients [(a): $\sigma^{\circ}{ }_{\mathrm{RH}}$; (b) $\sigma^{\circ}{ }_{\text {Rv }}$ ] vs wheat height

\subsection{Sensitivity analysis of Stokes parameters}

It is further analyzed the relationships between Stokes parameters and wheat height using regression approach. The scatterplots of Stokes parameters such are $\mathrm{g}_{0}, \mathrm{~g}_{1}, \mathrm{~g}_{2}$ and $\mathrm{g}_{3}$ with wheat height are given in Fig 5. The observed correlation coefficient and RMSE values for each case are given with the scatterplots. The Stokes fourth parameter $\left(\mathrm{g}_{3}\right)$, which represents the rotation direction of return signal, has shown higher correlation coefficient of 0.61 in comparison to $\mathrm{g}_{0}, \mathrm{~g}_{1}$ and $\mathrm{g}_{2}$ with $0.32,0.22$ and 0.05 respectively.

The scatterplot given in Fig 5d indicates that the return signal from wheat crop loses its degree of circularity towards left circular with increase in plant height. Earlier studies reported by many researchers (Srivastava et al., 2018a), clearly indicated that the Stokes first parameter $\left(\mathrm{g}_{0}\right)$, which represents the total intensity of signal, shown higher sensitivity towards paddy LAI. However go has shown good correlation with wheat height during stem elongation and milking stages, the inclusion of samples from ripening stage shown that this is less sensitive towards plant height. This indicates that the intensity of return signal can be used for plant height retrieval except during ripening stages, where plant height remains same but the water content in plants gets decrease. Since the plant water content highly correlates with the plant height before ripening, previous studies (Choudhury et al., 2007; Haldar et al., 2014) have observed the high sensitivity of backscattering coefficients with plant height. 


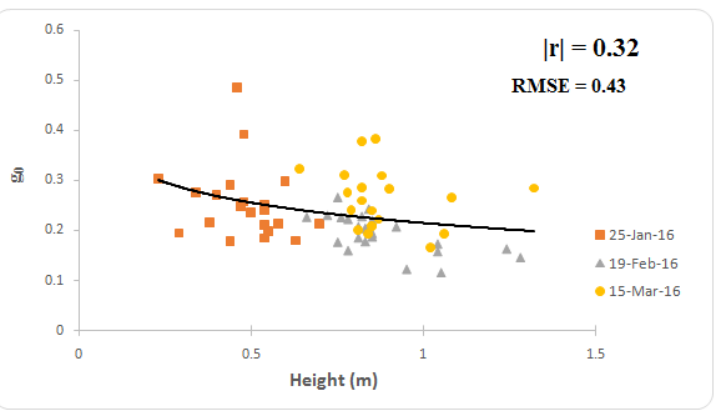

(a)

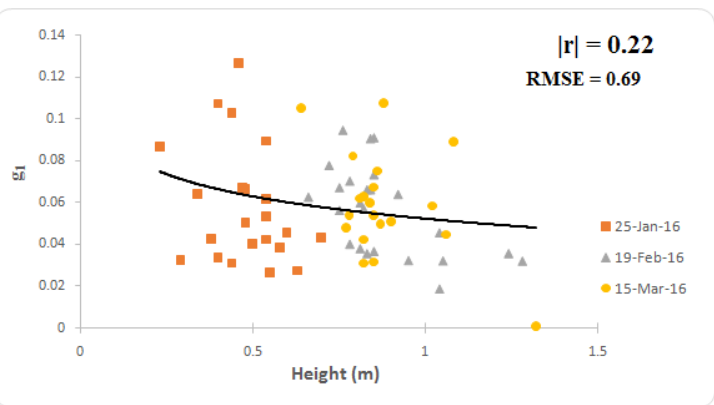

(b)

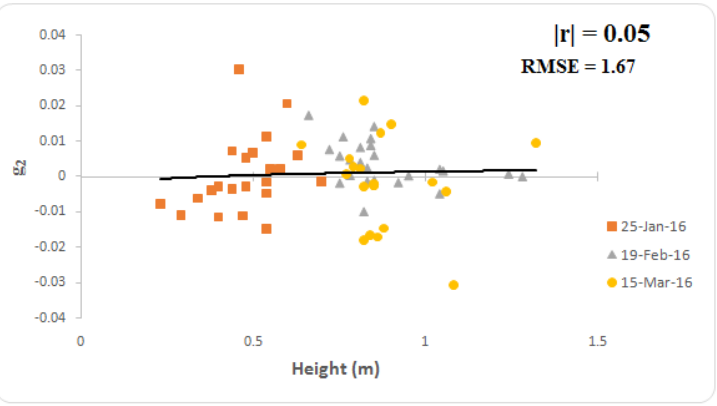

(c)

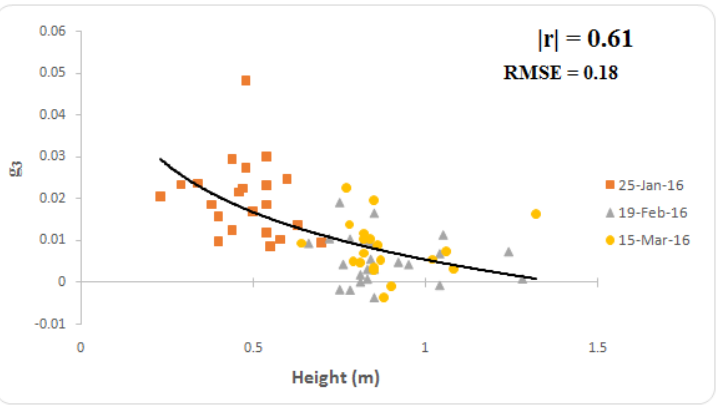

(d)

Figure 5. Scatterplot of Stokes parameters [(a): $g_{0} ;(b) g_{1} ;(c) g_{2}$; (d) $\left.g_{3}\right]$ vs wheat height

\subsection{Sensitivity analysis of $m-\chi$ decomposition derived parameters}

The scatterplots of decomposition parameters such are volume component, even bounce and odd bounce generated using $m-\chi$ space decomposition vs wheat height are given in Fig 6 . The correlation coefficient and RMSE (in $\mathrm{m}$ ) values as observed for each parameter are given with the scatterplots. Among the scattering decomposition parameters, odd bounce observed higher correlation coefficient than even bounce and odd bounce. Odd bounce which is primarily indicates the signal component directly interacts with the underneath soil through gaps, decreases with the crop growth, has shown higher sensitive towards plant height. However, in the case of plant height, fourth Stokes parameter $\left(\mathrm{g}_{3}\right)$ has shown higher correlation coefficient than decomposition parameters.

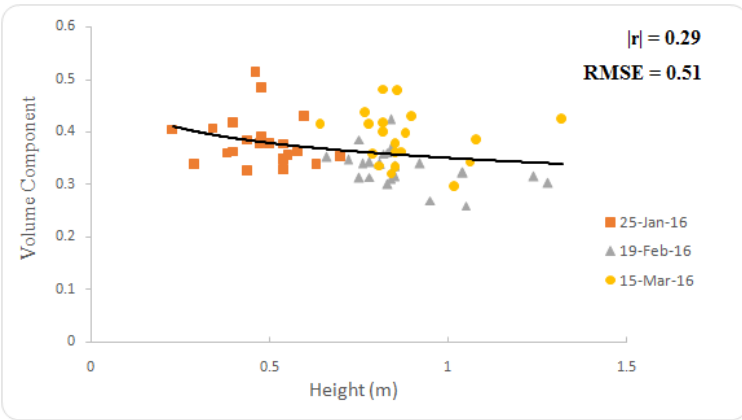

(a)

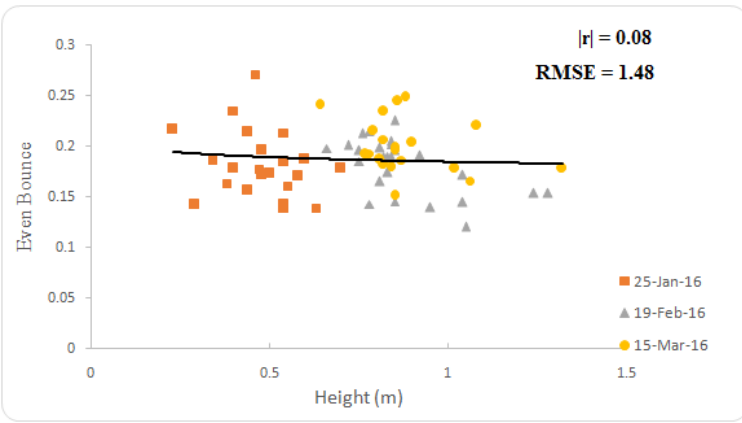

(b)

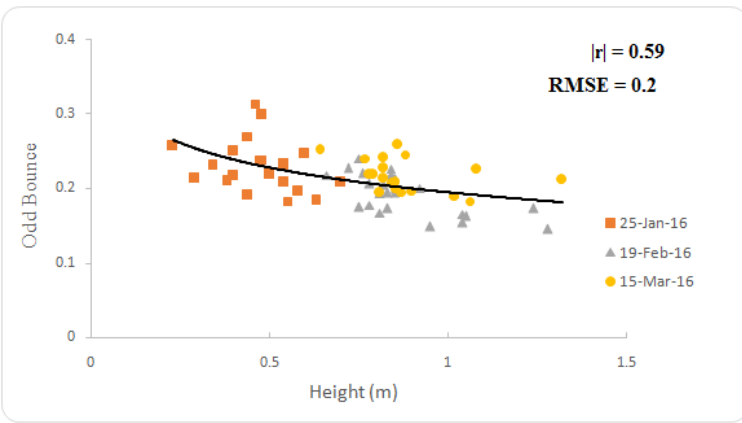

(c)

Figure 6. Scatterplot of Stokes parameters [(a): volume component; (b) even bounce; (c) odd bounce] vs wheat height

\section{CONCLUSIONS}

The sensitivity of parameters generated from RISAT-1 hybrid polarimetric SAR data (FRS-1 mode) towards wheat height have been explored using regression approach in this study. It is observed that the polarimetric parameters (Stokes parameters and $\mathrm{m}-\chi$ space decomposition) have shown higher sensitivity towards height than backscattering coefficients. The study results shown the highest correlation coefficient and lowest RMSE (in $\mathrm{m}$ ) for $\mathrm{g}_{3}$ with 0.61 and 0.18 respectively followed by odd bounce with 0.59 and 0.2 respectively. Since the odd 
bounce and $\mathrm{g}_{3}$ are highly dependent on the interaction with soil through gaps, it is identified as an indirect approach.

\section{REFERENCES}

Chakraborty, M., Panigrahy, S., Rajawat, A.S., Kumar, R., Murthy, T.V.R., Haldar, D., Chakraborty, A., Kumar, T., Rode, S., Kumar, H., Mahapatra, M., and Kundu, S., 2013. Initial results using RISAT-1 c-band SAR data. Current Science, 104(4), pp. 490-501.

Choudhury, I., Chakraborty, M., and Parihar, J.S., 2007. Estimation of rice growth parameter and crop phenology with conjunctive use of RADARSAT and ENVISAT. In: Proceeding of 'Enisat Symposium 2007', Montreux, Switzerland, pp. 1-6.

Curtis, B.C., Rajaraman, S., and MacPherson, H.G., 2002. Bread Wheat, Food and Agriculture Organization of the United Nations.

Doraiswamy, P.C., Moulin, S., Cook, P.W., and Stern, A., 2003. Crop yield assessment from remote sensing. Photogrammetric Engineering \& Remote Sensing, 69(6), pp. 665-674.

Erten, E., Lopez-Sanchez, J.M., Yuzugullu, O., and Hajnsek, I., 2016. Retreival of agricultural crop height from space: a comparison of SAR techniques. Remote Sensing of Environment, 187, pp. 130-144.

Haldar, D., Chakraborty, M., Manjunath, K.R., and Parihar, J.S., 2014. Role of polarimetric SAR data for discrimination/biophysical parameters of crops based on canopy architecture. In: The International Archives of the Photogrammetry, Remote Sensing and Spatial Information Sciences, Vol. XL-8, pp. 737-744.
Henderson, F.M., and Lewis, A.J., 1998. Principles and applications of imaging radar. Manual of remote sensing: Third edition, Volume 2.

Kumar, P., Prasad, R., Gupta, D.K., Mishra, V.N., Vishwakarma, A.K., Yadav, V.P., Bala, R., Choudhary, A., and Avtar, R., 2018. Estimation of winter wheat crop growth parameters using time series Sentinel-1A SAR data. Geocarto International, 33(9), pp. 942-956.

Macelloni, G., Paloscia, S., Pampaloni, P., Marliani, F., and Gai, M., 2001. The Relationship between the backscattering coefficient and the biomass of narrow and broad leaf crops. IEEE Transactions on Geoscience and Remote Sensing, 39(4), pp. 873-884.

Mishra, M.D., Patel, P., Srivastava, H.S., Shukla, A., Patel, P.R., Shukla, A.K., 2015. Approach for absolute radiometric calibration of RISAT-1 SAR data using standard target. International Journal of Remote Sensing \& Geoscience, 4(1), pp. 28-32.

Paloscia, S., Santi, E., Montomoli, F., Brogioni, M., Macelloni, G., Pampaloni, P., and Pettinato, S., 2014. The sensitivity of Cosmo-SkyMed backscatter to agricultural crop type and vegetation parameters. IEEE Journal of Selected Topics in Applied Earth Observations and Remote Sensing, 7(7), pp. 2856-2868.

Patel, P., and Srivastava, H.S., 2013. Ground truth planning for synthetic aperture radar (SAR): addressing various challenges using statistical approach. International Journal of Advancement in Remote Sensing, GIS and Geography, 1(2), pp. 1-17. 